\begin{tabular}{|c|c|}
\hline Title & Gapless phason in an antiferroelectric liquid crystal \\
\hline Author(s) & 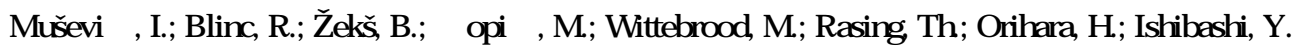 \\
\hline Citation & $\begin{array}{l}\text { Physical Review Letters, 71(8), 1180-1183 } \\
\text { https://doi.org/10.1103PhysRevLett.71.1180 }\end{array}$ \\
\hline Issue Date & $1993-08$ \\
\hline DOC URL & http:/hdl.handle.net/2115/50769 \\
\hline Rights & (c) 1993 A merican Physical Society \\
\hline Type & article \\
\hline File Information & Phys. Rev. Lett. 71.pdf \\
\hline
\end{tabular}

Instructions for use 


\title{
Gapless Phason in an Antiferroelectric Liquid Crystal
}

\author{
I. Muševič, R. Blinc, B. Žekš, and M. Čopič \\ J. Stefan Institute, Jamova 39, 61000 Ljubljana, Slovenia \\ M. M. Wittebrood and Th. Rasing \\ Research Institute for Materials and High Field Magnet Laboratory, University of Nijmegen, \\ Toernooiveld, 6525 ED Nijmegen, The Netherlands \\ H. Orihara and Y. Ishibashi \\ Synthetic Crystal Research Laboratory, School of Engineering, Nagoya University, Furo-cho, \\ Chikusa-ku, Nagoya 464-01, Japan \\ (Received 21 June 1993)
}

\begin{abstract}
The phason dispersion in the antiferroelectric $\mathrm{Sm}-C_{A}^{*}$ liquid crystalline phase of 4-(1-methylheptyloxycarbonyl)phenyl 4'-octyloxybiphenyl-4-carboxylate has been studied by quasielastic light-scattering experiments. The phason is found to be gapless as predicted for a Goldstone mode recovering the broken continuous symmetry. The dispersion has a minimum at $q=2 q_{c}$, where $q_{c}$ is the wave vector of the unperturbed $\mathrm{Sm}-C_{A}^{*}$ structure. These results are consistent with the alternating-tilt model of the antiferroelectric $\mathrm{Sm}-C_{A}^{*}$ phase.
\end{abstract}

PACS numbers: $61.30 . \mathrm{Gd}, 64.70 . \mathrm{Md}, 64.70 . \mathrm{Rh}$

The dynamics of liquid crystals is in certain respects richer than the dynamics of $3 \mathrm{D}$ periodic solids. In contrast to these systems, many liquid crystalline phases exhibit a continuous rotational symmetry, which can be broken at the transition to phases of lower symmetry. This should allow for the existence of symmetry recovering, zero frequency Goldstone modes, which are known to occur in particle physics and incommensurate systems. A particularly interesting Goldstone model, which is the subject of this study, should exist in antiferroelectric liquid crystals.

The antiferroelectric liquid crystalline $\mathrm{Sm}-C_{A}^{*}$ phase $[1,2]$ is characterized by an alternation of the tilt direction of the average molecular orientation and the direction of the in-plane spontaneous polarization $\mathbf{P}$ by nearly $\pm 180^{\circ}$ on going from one smectic layer to another [Fig. 1(a)]. Two neighboring layers thus form an antiferroelectric unit cell with two antiparallel electric dipoles and a zero value of the equilibrium electric polarization $\mathbf{P}_{0}(\mathbf{r})=\mathbf{P}_{i}+\mathbf{P}_{i+1}=0$. Because of chirality, the directions of the spontaneous tilt and the in-plane polarization slowly precess around the layer normal as one moves along the direction perpendicular to the smectic plane. This causes a small deviation from the $\pm 180^{\circ}$ alternation in the tilt between two consecutive layers and the formation of a modulated, helicoidal structure. Since the basic structural unit of the $\mathrm{Sm}-C_{A}^{*}$ phase are two neighboring layers, we have here in fact a double-twisted helicoidal structure, formed by two identical Sm- $C^{*}$ helices gearing into each other as shown in Fig. 1(b). The periodicity of this helical modulation is generally incommensurate to the basic antiferroelectric unit cell of the Sm- $C_{A}^{*}$ phase.

The theory of incommensurate systems [3] predicts the existence of a gapless phason mode in the incommensurate phase recovering the broken translational periodicity of the high temperature phase. In the antiferroelectric $\mathrm{Sm}-C_{A}^{*}$ phase, the phason mode represents the sliding, or what is equivalent, the slow rotation of the double twisted helicoidal modulation wave, restoring the symmetry, lost at the $\mathrm{Sm}-A \rightarrow \mathrm{Sm}-C_{A}^{*}$ transition. Since the $\mathrm{Sm}-A^{*}$ phase has the continuous $D_{\infty}$ symmetry, whereas the symmetry of the Sm- $C_{A}^{*}$ phase is discrete, the symmetry recovering phason is here predicted to be a truly gapless Goldstone mode.

Whereas the presence of acousticlike phason modes in structurally incommensurate systems has been by now clearly demonstrated [4], no truly gapless phason branch has been observed so far in systems with discrete lattice

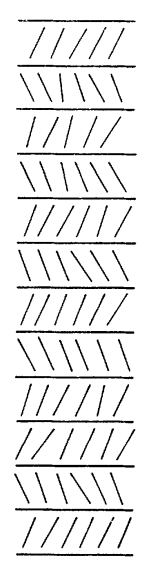

a)

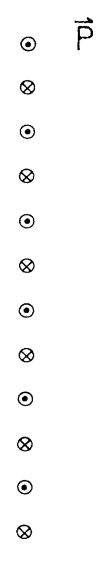

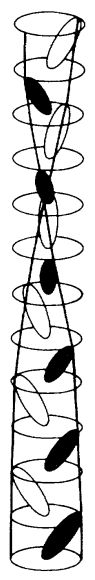

b)
FIG. 1. (a) The alternating tilt model of the homogeneous antiferroelectric Sm- $C_{A}^{*}$ phase of liquid crystals and (b) the antiferroelectric double helix. 
symmetry, and the question of the possible existence of such modes is still open. The only gapless phason dispersion observed so far has been in ferroelectric liquid crystals [5], where the basic structure is quasicontinuous, rather than discrete, as the one-dimensional smectic density modulation is only weakly coupled to the ferroelectric order parameter and the polarization and the tilt change only infinitesimally on going from one smectic layer to another. A phason dispersion measurement has been also performed in the $\mathrm{Sm}-C_{\gamma}^{*}$ phase [6], where, however, the results seemed to show a finite value of the phason relaxation frequency at zero scattering wave vector, $q=0$.

Here we report the results of a quasielastic light scattering phason dispersion study of the antiferroelectric Sm- $C_{A}^{*}$ phase using the backwards scattering geometry. The dispersion relation of the phason modes in the antiferroelectric phase is found to be gapless, as expected for a Goldstone mode, restoring the broken continuous symmetry of the $\mathrm{Sm}-A$ phase at the $\mathrm{Sm}-A \rightarrow \mathrm{Sm}-C^{*}$ or $\mathrm{Sm}-A \rightarrow \mathrm{Sm}-C_{A}^{*}$ phase transition points. The dispersion relation for the phason modes show a minimum at $2 q_{c}$, where $q_{c}=2 \pi / p$ and $p$ is the period of the helix. This result is clearly compatible with the selective reflection experiments by Chandani et al. [1] and the proposed alternating tilt structure of the $\mathrm{Sm}-C_{A}^{*}$ phase. The form of the dielectric tensor, which is compatible with both these experiments can be obtained as a space average of the dielectric tensors to two oppositely tilted ferroelectric $\mathrm{Sm}-\mathrm{C}^{*}$ layers. It should be stressed that a previous attempt to study the phason dynamics in the $\mathrm{Sm}-C_{A}^{*}$ phase using the forward scattering geometry [6] did not reveal the phason dispersion along $q_{z}$. The phason frequency obtained was of the order of $250 \mathrm{kHz}$ and agrees with our values, extrapolated to the Brillouin zone center, $q=0$.

The thermodynamic properties and phase transitions between the antiferroelectric $\mathrm{Sm}-C_{A}^{*}$ phase and the related ferri-, ferro-, and paraelectric phases have been theoretically analyzed by Orihara and Ishibashi [7] and later by Žekš, Blinc, and Čepič [8] within the framework of a Landau theory. The order parameters of the phase transitions between these phases have been conveniently chosen as linear combinations of the tilt vectors $\xi_{i}$ and $\xi_{i+1}$ in the two neighboring smectic layers $i$ and $i+1$,

$$
\begin{aligned}
& \xi_{f}=\frac{\xi_{i}+\xi_{i+1}}{2}, \\
& \xi_{a}=\frac{\xi_{i}-\xi_{i+1}}{2},
\end{aligned}
$$

thus describing the ferroelectric $\left(\xi_{f}\right)$ and antiferroelectric $\left(\xi_{a}\right)$ ordering, respectively. By writing down scalar invariants of the two order parameters [8] with respect to the $D_{\infty}$ symmetry of the paraelectric $\mathrm{Sm}-A$ phase, one obtains by minimization regions of stability of the para-, ferri-, ferro-, and antiferroelectric phases. These phases are characterized by different equilibrium values of the order parameters $\xi_{f}^{0}$ and $\xi_{a}^{0}$. For example, in the paraelectric $\mathrm{Sm}-A$ phase $\xi_{f}^{0}=\xi_{a}^{0}=0$, in the ferroelectric phase $\xi_{f}^{0} \neq 0$ and $\xi_{a}^{0}=0$, in the ferrielectric phase $\xi_{a}^{0} \neq 0$ and $\xi_{f}^{0} \neq 0$, whereas in the antiferroelectric phase $\xi_{a}^{0} \neq 0$ and $\xi f=0$.

The spectrum of elementary excitations of the director-polarization field in the antiferroelectric liquid crystal has been first discussed by Žekš, Blinc, and Čepič [8]. The onset of antiferroelectric order at the phase transition point is accompanied by the slowing down of a soft mode, that has an antiferroelectric (nonpolar) character. Whereas in ferroelectric liquid crystals the soft mode represents the condensation of a plane wave excitation with a wave vector $q_{c}$ which is close to the center of the Brillouin zone, as $q_{c}=2 \pi / p$, where $p$ is the period of the helical structure, the antiferroelectric soft mode here represents the condensation of a plane wave with the wave vector $q_{d}$ near the edge of the Brillouin zone. Here $q_{d}=\pi / d \gg q_{c}$, where $d$ is the smectic interlayer distance, and the Brillouin zone (Fig. 2) of the homogeneous antiferroelectric phase is $(-\pi / d, \pi / d)$ and reflects the antiparallel orientation of the tilt vectors in two neighboring smectic layers.

The dynamics of the phason modes in the $\mathrm{Sm}-C_{A}^{*}$ phase can be approximately derived from the phase dependent part of the nonequilibrium free energy density in the constant amplitude approximation

$$
g(z, t)=-\Lambda_{a} \theta_{0}^{2}\left(\frac{\partial \Phi(z, t)}{\partial z}\right)+\frac{1}{2} K_{3 a} \theta_{0}^{2}\left(\frac{\partial \Phi(z, t)}{\partial z}\right)^{2},
$$

which is similar to the free-energy expansion for the phason excitations in the ferroelectric $\mathrm{Sm}-C^{*}$ phase [5]. Here, $\Lambda_{a}$ and $K_{3 a}$ are Lifshitz and torsional elastic constants, associated with the antiferroelectric order parameter $\boldsymbol{\xi}_{a}$, which is expressed as

$$
\xi_{a}(z, t)=\theta_{0}(\cos \Phi(z, t), \sin \Phi(z, t)) .
$$

Following the Landau-Khalatnikov equations of motion

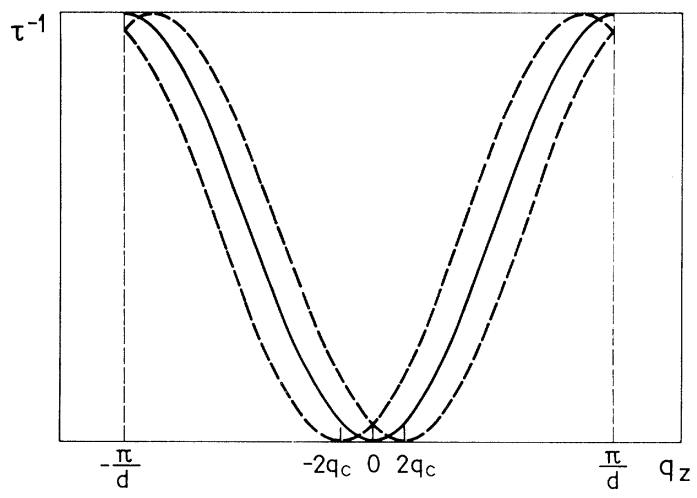

FIG. 2. Schematic Brillouin zone for phase excitations in the homogeneous $\mathrm{Sm}-C_{A}^{*}$ phase. The dispersion relation for the case of the helicoidal modulation is shown by the dashed line. 
for the nonequilibrium phase profile $\Phi(z, t)=q_{c} z$ $+\delta \Phi(z, t)$ in the unperturbed $\mathrm{Sm}-C_{A}^{*}$ phase, one obtains overdamped plane-wave solutions for the phase excitations

$$
\delta \Phi_{q}(z, t) \propto e^{-t / \tau(q)} \cos (q z+\phi),
$$

where $\phi$ is arbitrarily chosen. The corresponding phason dispersion relation is gapless and parabolic:

$$
\tau^{-1}(q)=\frac{K_{3 a}}{\gamma} q^{2}
$$

It should be noted that in view of the relation $\Phi(z, t)$ $=q_{c} z+\delta \Phi(z, t)$ the above expressions are written in a frame, which rotates together with the helix, and that in the laboratory frame the phason dispersion has a minimum at finite $q$. One should also mention that in the low-frequency and long-wavelength limit $q \rightarrow 0$, the phason excitations in the $\mathrm{Sm}-C_{A}^{*}$ phase are nonpolar, i.e., there is no fluctuating electric dipole moment, $\delta \mathbf{P}(z, t)$ $=0$. This has the consequence, that contrary to the case of ferroelectric liquid crystals, antiferroelectric eigenmodes with $q \rightarrow 0$ cannot contribute to the linear response of the $\mathrm{Sm}-C_{A}^{*}$ phase in the dielectric experiment. On the other hand, these eigenmodes give rise to strong fluctuations of the dielectric tensor and should be observable in quasielastic light scattering experiments.

The inhomogeneous part of the dielectric tensor of the $\mathrm{Sm}-C_{A}^{*}$ phase [9], which is responsible for the quasielastic light scattering, is in the limit of small tilt angle and small amplitudes of phase excitations directly proportional to the phase excitation $\delta \Phi_{q}(z, t)$ :

$$
\begin{aligned}
\delta \epsilon(z, t)= & \frac{1}{2}\left(\epsilon_{3}-\epsilon_{1}\right) \theta_{0}^{2}\left[\begin{array}{ccc}
-\sin \left(2 q_{c} z\right) & \cos \left(2 q_{c} z\right) & 0 \\
\cos \left(2 q_{c} z\right) & \sin \left(2 q_{c} z\right) & 0 \\
0 & 0 & 0
\end{array}\right] \\
& \times \delta \Phi_{q}(z, t) .
\end{aligned}
$$

Here, we have taken for simplicity the uniaxial form of the dielectric tensor for optical frequencies, $\epsilon_{1}=\epsilon_{2} \neq \epsilon_{3}$, where $\epsilon_{i}$ are the corresponding eigenvalues, defined in Ref. [9]. From the above expression one observes that the phason excitation $\delta \Phi_{q}(z, t)$ with the wave vector $q$ is observable via fluctuations of the dielectric tensor as an excitation with the wave vector $q \pm 2 q_{c}$. The dispersion relation for the phason excitations in the $\mathrm{Sm}-C_{A}^{*}$ phase has in the laboratory frame a minimum at the scattering wave vector $q_{s}=2 q_{c}$ :

$$
\tau^{-1}(q)=\frac{K_{3 a}}{\gamma}\left(q \pm 2 q_{c}\right)^{2} .
$$

The same result is obtained from a lattice model [10] where the discrete structure of the $\mathrm{Sm}-C_{A}^{*}$ phase is explicitly taken into account.

The experiment was performed in the $\mathrm{Sm}-C_{A}^{*}$ phase of 4-(1-methylheptyloxycarbonyl)phenyl 4'-octyloxybiphenyl-4-carboxylate (MHPOBC), slightly below the
$\mathrm{Sm}-C_{\gamma} \rightarrow \mathrm{Sm}-C_{A}^{*}$ phase transition point. We have used well aligned homeotropic samples of thickness $50 \mu \mathrm{m}$ in an experimental arrangement, described elsewhere [11]. In view of the rather small values of the period of the helix near the $\mathrm{Sm}-C_{\gamma} \rightarrow \mathrm{Sm}-C_{A}^{*}$ phase transition point in this substance, which corresponds to a large critical wave vector $q_{c}$, we have decided to measure the phason dispersion relation in a backscattering, depolarized geometry, as shown in the inset to Fig. 3. The light-collecting optics was positioned at a small angle $\left(\approx 2^{\circ}\right)$ with respect to the light reflection from the samples surface, thus assuring a heterodyne detection regime. This small deviation of the detector angle from direct reflection introduces a small transverse component $q_{x}$ of the wave vector which has been treated as a small perturbation to the dispersion relation [12].

The dispersion relation for phase excitations propagating along the helical axis of the $\mathrm{Sm}-C_{A}^{*}$ phase of MHPOBC as obtained by the quasielastic light scattering in a backscattering geometry is shown in Fig. 3. The solid line represents the best fit to Eq. (7) giving $K_{3 a} / \gamma$ $=0.58 \times 10^{3} \mu \mathrm{m}^{2} \mathrm{~s}^{-1}$. From the contribution of the transverse component $q_{x}$ of the wave vector to the dispersion relation we can estimate $K+a / \gamma \approx 1 \times 10^{3} \mu \mathrm{m}^{2} \mathrm{~s}^{-1}$. Here $K+a=\frac{1}{2}\left(K_{S}+K_{B}\right)$ is an effective transverse elastic constant [12], $K_{S}$ and $K_{B}$ are splay and bend elastic constants, respectively, and $\gamma$ is the corresponding viscosity. As one can see from Fig. 3, the phason dispersion is gapless within the limits of the experimental error and centered at the scattering wave vector $q_{s}=20.3 \mu \mathrm{m}^{-1}$. For $q_{s}=2 q_{c}$ this corresponds to a helix with a wave vector

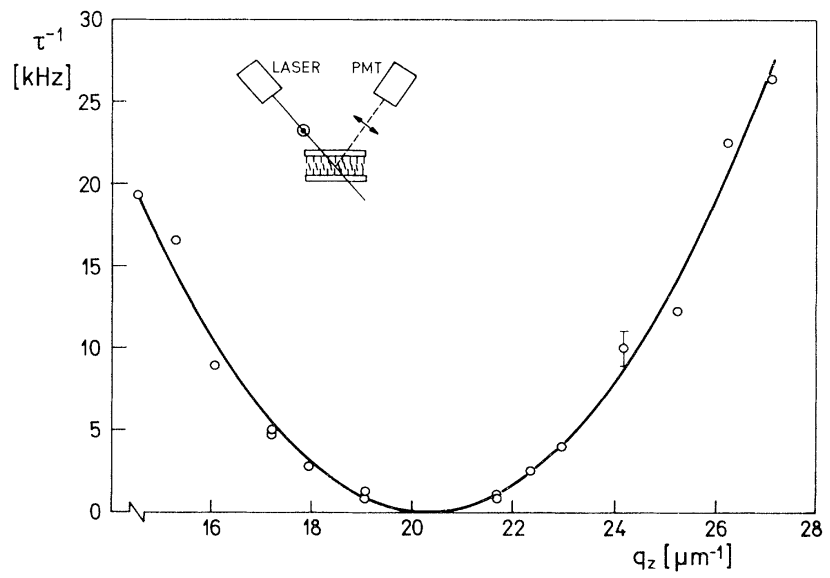

FIG. 3. Phason dispersion in the $\mathrm{Sm}-C_{A}^{*}$ phase of MHPOBC, $0.1 \mathrm{~K}$ below the $\mathrm{Sm}-C_{\gamma} \rightarrow \mathrm{Sm}-C_{A}^{*}$ transition. The solid line represents the best fit to the Eq. (7) with $K_{3 a} / \gamma=0.58 \times 10^{3} \mu \mathrm{m}^{2} \mathrm{~s}^{-1}$ and $K+a / \gamma=1 \times 10^{3} \mu \mathrm{m}^{2} \mathrm{~s}^{-1}$. The inset shows the scattering geometry. The magnitude of the scattering wave vector $q_{s}$ along the $z$ direction was calculated according to the uniaxial optical model of the $\mathrm{Sm}-C_{A}^{*}$ phase, with refractive indices $n_{o}=1.5$ and $n_{e}=1.625$, according to Ref. [18]. 
$q_{c}=10.15 \mu \mathrm{m}^{-1}$ or a period of $0.62 \mu \mathrm{m}$. The period of the helicial structure in the $\mathrm{Sm}-C_{A}^{*}$ phase of MHPOBC can also directly be obtained by the selective reflection method as has been measured by Chandani et al. [1]. From the position of the selective reflection peak near the $\mathrm{Sm}-C_{\gamma} \rightarrow \mathrm{Sm}-C_{A}^{*}$ phase transition point, taking into account an average refractive index of the $\mathrm{Sm}-C_{A}^{*}$ phase of MHPOBC and assuming a form of the dielectric tensor given by Eq. (6), one obtains the period of the helix of MHPOBC $p=0.6 \mu \mathrm{m}$, which is in excellent agreement with experimental observations.

The value of $K_{3 a} / \gamma$, as obtained from Fig. 3 is within an order of magnitude the same as that obtained in quasielastic light scattering experiments in the ferroelectric liquid crystals 4-(2'-methylbutyl)-phenyl 4 '- $n$-octylbiphenyl-4-carboxylate (CE-8) and $p$-decyloxybenzylidene- $p$ '-amino-2-methylbutyl cinnamate (DOBAMBC) $[5,12]$. In those experiments, one would usually observe a ratio $K_{+} / \gamma$ which is 1 order of magnitude larger. This is in contrast with the situation in MHPOBC, where $K+a / \gamma$ is only a factor of 2 larger than $K_{3 a} / \gamma$. Such a low value of $K_{+a} / \gamma$ in the antiferroelectric Sm- $C_{A}^{*}$ could be a result of the absence of a spontaneous polarization $\mathbf{P}(\mathbf{r}, t)$ in this phase. It was argued [13-16] and recently observed [17] that the presence of a fluctuating dipole field $\mathbf{P}(\mathbf{r}, t)$ significantly influences the magnitude of the transverse (or in-plane) elastic constant $K+$ due to bend fluctuations of the C-director field, which represent splaylike fluctuations of the spontaneous polarization field $\mathbf{P}(\mathbf{r}, t)$. This results in the appearance of a fluctuating space charge density $\rho(\mathbf{r}, t)=-\boldsymbol{\nabla P}(\mathbf{r}, t)$ and renormalizes bend elastic constant $K_{B}$ due to electrostatic self-energy of charge distribution $[16,17]$.

The value of $K_{3 a} / \gamma$, as obtained from Fig. 3, can be compared to the phason relaxation rates, as obtained by Sun, Orihara, and Ishibashi [6] in the Sm- $C_{A}^{*}$ phase of MHPOBC at $q_{s} \rightarrow 0$. Their forward scattering geometry allowed only for the determination of phason relaxation rates in the limit of small scattering wave vectors, $\mathbf{q}_{s} \rightarrow 0$. In this limit they obtain relaxation rates in the $\mathrm{Sm}-C_{A}^{*}$ phase of MHPOBC of the order of $200-300 \mathrm{kHz}$. By extrapolating our data in Fig. 3 to $\mathbf{q}_{s}=0$, we obtain phason relaxation rates of the order of $\tau^{-1}(q=0) \approx 250 \mathrm{kHz}$, which is in excellent agreement with the results of Sun, Orihara, and Ishibashi.

In conclusion, we report for the first time the observation of a phason dispersion in the $\mathrm{Sm}-C_{A}^{*}$ phase of an antiferroelectric liquid crystal. The dispersion relation for phason excitations, propagating along the helical axis of the $\mathrm{Sm}-C_{A}^{*}$ phase, is gapless, as expected for a Goldstone mode restoring the broken continuous symmetry of the $\mathrm{Sm}-A$ phase. It has a minimum at the wave vector $2 q_{c}$, where $q_{c}$ is the wave vector of the unperturbed $\mathrm{Sm}-C_{A}^{*}$ structure. This observation is consistent with the proposed form of the dielectric tensor in the $\mathrm{Sm}-C_{A}^{*}$ phase and the alternating-tilt model of the antiferroelectric $\mathrm{Sm}-C_{A}^{*}$ phase of liquid crystals and seems to be the first demonstration of the gapless nature of the phason mode in any incommensurate system with discrete lattice symmetry.

The authors thank Dr. I. Kawamura, Showa Shell Sekiyu Co., Ltd., for supplying MHPOBC samples.

[1] A. D. L. Chandani, E. Gorecka, Y. Ouchi, H. Takezoe, and A. Fukuda, Jpn. J. Appl. Phys. 28, L1265 (1989).

[2] Ch. Bahr and D. Fliegner, Phys. Rev. Lett. 70, 1842 (1993).

[3] P. Bak, Rep. Prog. Phys. 45, 587 (1982).

[4] See, for example, Incommensurate Phases in Dielectrics, edited by R. Blinc and A. P. Levanyuk (North-Holland, Amsterdam, 1986), Vol. 2.

[5] I. Muševič, R. Blinc, B. Žekš, C. Filipič, M. Čopič, A. Seppen, P. Wyder, and A. Levanyuk, Phys. Rev. Lett. 60, 1530 (1988). See also I. Muševič, B. Žekš, R. Blinc, H. A. Wierenga, and Th. Rasing, Phys. Rev. Lett. 68, 1850 (1992).

[6] H. Sun, H. Orihara, and Y. Ishibashi, J. Phys. Soc. Jpn. 60, 4175 (1991).

[7] H. Orihara and Y. Ishibashi, Jpn. J. Appl. Phys. 29, L115 (1990).

[8] B. Žekš, R. Blinc, and M. Čepič, Ferroelectrics 122, 221 (1991).

[9] I. Muševič, B. Žekš, R. Blinc, and Th. Rasing, Phys. Rev. E 47, 1094 (1993).

[10] B. Žekš et al. (to be published).

[11] I. Muševič, B. Žekš, R. Blinc, H. A. Wierenga, and Th. Rasing, Physica (Amsterdam) 177B, 511 (1992).

[12] I. Drevenšek, I. Muševič, and M. Čopič, Phys. Rev. A 41, 923 (1990).

[13] C. Y. Young, R. Pindak, N. A. Clark, and R. B. Meyer, Phys. Rev. Lett. 40, 773 (1978).

[14] C. Rosenblatt, R. Pindak, N. A. Clark, and R. B. Meyer, Phys. Rev. Lett. 42, 1220 (1979).

[15] J. F. Palierne, Phys. Rev. Lett. 56, 1160 (1986).

[16] K. Okano, Jpn. J. Appl. Phys. 25, L846 (1986).

[17] Min-Hua Lu, Karl A. Crandall, and Charles Rosenblatt, Phys. Rev. Lett. 68, 3575 (1992).

[18] E. Gorecka, A. D. L. Chandani, Y. Ouchi, H. Takezoe, and A. Fukuda, Jpn. J. Appl. Phys. 29, 131 (1990). 